\title{
Shooting for the Moon
}

\author{
The UN Sustainable Development Goals aim to solve the most serious global economic, societal and \\ environmental issues. The microbiology community is in a unique position to help tackle this ambitious \\ agenda for sustainable development.
}

Head to any British cinema at the moment and you may well find yourself transported back to the 1990s as 'Wannabe' blasts out in surround sound during the previews. No, there hasn't been a Spice Girls movie remake (sorry). Instead, this music video delivers a far more serious message about gender equality, education and equal pay for equal work. Launched by Project Everyone (https://www.project-everyone.org/), this nostalgic yet highly topical video promotes some of the Sustainable Development Goals (SDGs) adopted by the UN sustainability agenda in 2015. The SDGs are a suite of 17 overall goals with 169 (yes, 169) targets aimed at improving life on Earth, specifically to end poverty, protect the planet, and ensure prosperity for $\mathrm{all}^{1}$. Few would disagree with the SDG focus on the world's most serious problems, and credit should be given for their lofty ambitions; the first goal is to, quite simply, "End poverty in all its forms everywhere". The SDGs are ambitious, admirable, yet more than just a little hopeful, but are they in any way achievable by their 2030 deadline, and if so how can microbiologists contribute?

The SDGs follow and expand on a previous set of Millennium Development Goals (MDGs) set out in 2000. This framework of 8 goals and 21 targets pales in comparison to the much expanded SDG list, but there was a familiar ambition to create a global partnership to reduce extreme poverty with a series of timelimited targets. However, the MDGs were not universally achieved; in the words of Ban Ki-moon, the UN secretary general, "inequalities persist and ... progress has been uneven"2. The SDGs were designed to account for some of the shortcomings of the MDGs, which did not seek to tackle the 'root causes' of poverty and overlooked the integrated nature of sustainable development ${ }^{2}$. However, obstacles such as lack of funding, bad governance, and corruption (among others) are still present, rightly leading to questions about how SDGs could be achieved in just 15 years. In an attempt to solve everything, there is a danger of diluting funding, effort and focus. The UN disagrees with these concerns, having learned from missed opportunities during the MDG era. By having an extensive list of targets, the hope is that there will be a shift towards an integrated approach to tackling societal issues, exemplified in goal 17 , which explicitly aims to strengthen the "global parhealthtnership for sustainable development". We are being encouraged to ask how big issues such as poverty, education, sustainability and food security can be tackled together, maximizing impact whilst managing costs efficiently. Knock-on effects of potential policies and interventions should be considered. For example, by empowering people to use condoms in HIV endemic areas, not only will the burden of infectious disease on healthcare services and patients be alleviated, but it will also have knock-on economic and societal benefits that will reduce poverty. Take a browse of the Internet and it's easy to stumble across discussions about the achievability of the SDGs. The consensus tends to be that yes, some of the goals are achievable but only if we can address an unsurprising list of caveats such as the need to reduce corruption, improve political will, empower communities, embrace technological advance, and so forth. It is clear that the obstacles that so dogged the MDGs are not gone, yet there remains a palpable feeling of hope.

So, what can we do? In fact, the microbiological community is well-placed to take a lead in tackling many of the SDG targets: beating infectious disease, provision of clean water, food security, maintenance of biodiversity in the oceans and on land, and uptake of green energy such as biofuels. The multi-disciplinary nature of microbiological research means that we are also used to taking an integrated approach to answering questions and solving problems ${ }^{3}$. Technological advance, the evolution of ideas, cooperative behaviour and sharing of resources are common-place in the microbiology lab (for both the scientists and the microorganisms). As an example, recent culture-independent analyses of microbial communities have highlighted the vastness of microbial diversity ${ }^{4}$. This insight would not have been possible without technological developments, publicly available data and input from diverse disciplines such as computer and environmental sciences, and the wider impacts cannot be realized without considering the biogeochemistry and conservation fields.

The goals and targets also provide a framework for considering the impact of research, a factor becoming more and more important for funding bodies around the world. Historically, most scientific research has been confined to the upper echelons of society. But times are changing - social media, television, public events and global connectivity have made the latest scientific discoveries more readily available than ever before, opening up opportunities for scientific engagement in an attempt to drive change. With some lateral thinking, all the SDGs are relevant to microbiology and simple engagement. Sharing a \#lobalGoals selfie whilst collecting samples, or adding your research location to the Global Goals app (http://www.globalgoals.org/ tell-everyone), will help to raise awareness of the SDGs. Furthermore, collaboration with policy-makers, funders and nongovernmental organizations will help to maximize research impact within the wider SDG context.

Of course, microbiologists have been working for decades (if not centuries) on the problems highlighted by the SDGs, and will need to continue to do so once 2030 has come and gone. The SDGs are worthy and ambitious aims, yet even with fortuitous tailwinds, achieving more than a few of the lower-hanging targets will be challenging. Nevertheless, not even attempting to solve these intractable problems is not an option, and ambitious targets are sure to drive innovation in an attempt to get close to success. The SDGs may seem like we are shooting for the Moon, but even if we miss, the journey will see us land among the stars, improving the lives of many millions of people along the way.

\footnotetext{
References

1. Sustainable Development Goals United Nations (2016); http://www.un.org/sustainabledevelopment/ sustainable-development-goals/

2. The Millennium Development Goals Report 2015 (United Nations, 2015); http://www.un.org/millenniumgoals/2015_MDG_Report/ pdf/MDG\%202015\%20rev\%20(July\%201).pdf

3. Blainey, P. C. Nat. Microbiol. 1, 16118 (2016).

4. Hug, L. A. et al. Nat. Microbiol. 1, 16048 (2016).
} 